\title{
Design and Research of HMI Based on the Qt for CNC System
}

\author{
Ruirui Sun ${ }^{1, a}$, Guanghe Cheng ${ }^{1, b}$, Xiang Sun $^{1, c}$ \\ ${ }^{1}$ Shandong Computer Science Center (National Supercomputer Center in Jinan), Shandong \\ Provincial Key Laboratory of Computer Networks, Jinan 250014, Shandong, China \\ aemail: sunrr@sdas.org, bemail:chenggh@sdas.org, 'email:sunx@sdas.org
}

Keywords: CNC system; HMI; WinCE; Qt

\begin{abstract}
Development of NC system HMI requirements, construction and development of the numerical control system human-machine interface system based on the AM3352. WinCE system, introduced in Qt language design and development method of embedded numerical control system human-machine interface. Design of human-machine interface based on Qt and related applications, including the overall framework design, the g-code editor design, design and graphic simulation of the main part of the study file manager. For G02 and G03 graphic simulation paper also presents a more detailed algorithm steps. Experiment and test results showed that the CNC system is the system of human-computer interaction and control requirements.
\end{abstract}

\section{Introduction}

With the rapid development of computer technology, embedded technology has now become a hot new trend. CNC system as a special-purpose computer systems with industrial control functions as embedded technologies become more sophisticated, more and more to the development of networked, intelligent, and miniaturized. For fast developing embedded CNC system control program, breaking away from the traditional poor interface versatility, stability, software development lifecycle and other shortcomings, people come to look into research on human-machine interface based on embedded system, particularly with respect to different embedded operating systems needed a cross-platform development language, so this article uses the Qt cross-platform development language as the design and development of open CNC system of a human-machine interface development languages. Research and development of open CNC system for promoting the development of industrial automation plays a huge role [1] [2]. Based on EPC-9200 Control Board +WinCE for the development of embedded NC system HMI platform using Qt as a development language.

\section{Qt introduction and development environment to build}

Qt introduction. Qt is a cross-platform C++ graphical user interface library, by Norway TrollTech Company, Qt supports all UNIX systems, including Linux, of course, also supports WinNT/WinCE, Win95/98 platform. Basically, Qt has like Openwin, GTK, MFC, VCL, ATL this kind of thing, but Qt has the following advantages: (1) a good cross-platform features, (2) object-oriented, and (3) rich API and (4) support 2D/3D graphics rendering, supports OpenGL and (5) a lot of development document (6) XML support[3][4].

Development environment to build. CNC system based on WinCE development needs based on WinCE system PC development environment, that is, build "VS+Qt+SDK" development environment. This specific development environments are as follows: hardware includes installing 
WIN7 PC machine, pre-installed Windows Embedded CE6.0 EPC-9200I-W industrial control panel software, including Visual Studio2008, WinCE6.0_Standard_SDK, Microsoft ActiveSync4.5. And then Qt4.8 (higher or just need a later version of VS) compiled class library configuration to VS2008, which can develop Qt applications on VS2008, environment build complete.

\section{CNC system}

CNC system Functional Requirements. According to actual needs, design block diagram of the main interface of the CNC system is constructed, as shown in Figure 1. CNC main interface is divided into coordinates, program editing, paranoid setting, processing, monitoring, maintenance, diagnosis, parameter setting, system, this 8 module as a first layer [5]. Through the lower edge of the display 8 buttons for switching modules and commands. Which, coordinates module main is responsible for coordinates switch, and clear, function; program edit module main achieved processing program of edit, and file management and graphics simulation, function part; partial reset set module main achieved artifacts coordinate system set, and props set, and on knife, function; processing monitoring module main is graphics simulation displayed and processing records, and clear time cumulative, and graphics adjustment, function; maintenance module main is including alert displayed, and network set and information backup, function; Diagnostic functions mainly on fast diagnosis of system operation, including the PLC status, system information, sharing information, program variables and operating records; parameters including the parameter matrix, application parameters, parameters and compensation table data system module was introduced to system manufacturers and system profile.

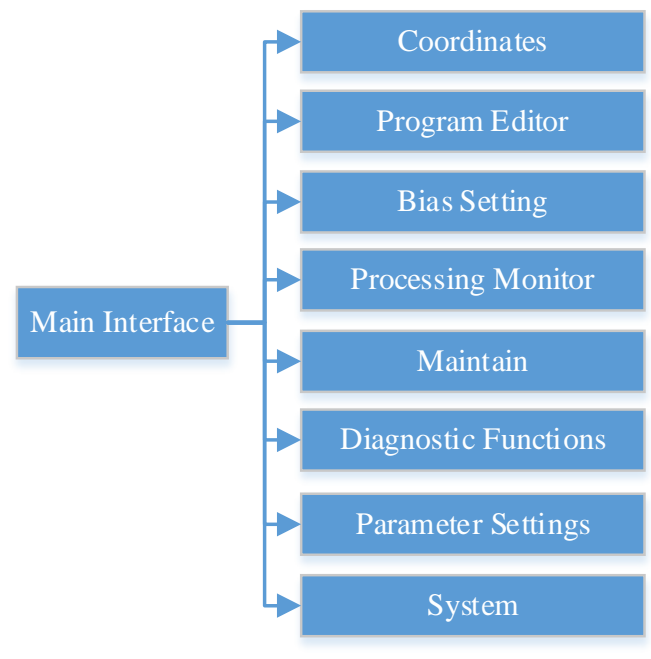

Fig.1. CNC Main Interface Diagram 
Tab.1. Function Name

\begin{tabular}{|c|c|c|c|}
\hline One Function & Program Name & Two Function & Program Name \\
\hline \multirow[t]{3}{*}{ Coordinates } & Coordinate() & 1 Coordinates Switch & SwitchCoordinate() \\
\hline & & 2ClearRelativeCoordinates & ClearRelaCoordinate() \\
\hline & & $\cdots$ & $\ldots$ \\
\hline \multirow[t]{5}{*}{ Program Editor } & ProgramEditor() & 1File Management & FileManagement() \\
\hline & & 2 Graphic Simulation & SimulateGraph() \\
\hline & & 3 Delete Row & DeleteRow() \\
\hline & & 4 Copy & Сору() \\
\hline & & 5 Paste... & Paste ()$\ldots$ \\
\hline \multirow[t]{2}{*}{ Bias Setting } & SetOffset() & 1Workpiece Coordinate & WorkpieceCoordnate() \\
\hline & & 2Tool Setting... & SettingTool()... \\
\hline \multirow[t]{5}{*}{ ProcessingMonitor } & Coordinate() & 1 Graphic Analog Display & DisplayAnalogGraph() \\
\hline & & $2 \mathrm{MPI}$ & MPI() \\
\hline & & 3 Processing Setting & SetManuInformation() \\
\hline & & 4Processing & ProcessRecordForm()... \\
\hline & & Table... & \\
\hline \multirow[t]{3}{*}{ Maintain } & Maintenance() & 1Alarm Display & Alarm() \\
\hline & & 2System Settings & SystemSettings() \\
\hline & & 3Data Backup... & DataBackup()... \\
\hline Diagnostic & DiagnosticFunction() & 1 PLC Status & PLCStatus() \\
\hline \multirow[t]{2}{*}{ Functions } & & 2Shared Data & SharedData() \\
\hline & & 30peration Record... & OperationRecord()... \\
\hline \multirow[t]{2}{*}{ Parameter Settings } & ParameterSetting() & 1Parameter Summary Table & ParameterSumTable() \\
\hline & & 2 Application Parameters... & ApplicationPara()... \\
\hline System & System() & $\ldots$ & $\ldots$ \\
\hline
\end{tabular}

Module interface definition and communication mechanism. In order to develop cooperation and exchanges in the late advance the module functions defined according to certain rules, as shown in table 1. Once defined, between the various functional modules can be called directly through the class name or function name, and reduction of multiple-person cooperation programming naming inconsistency problem.

Qt signals and slots of communication mechanisms between the modules is through "QPushButton" implemented by the class, the jump function using "clicked ()" slot is to be achieved. To coordinate the menu, for example, in the main window in the header file (MainWindow.h) contains the coordinates of the module header file (coordinatedialog.h) and in main window class defines the coordinate class (CoordinateDialog) to a coordinate object (coordinate), and then in the main window within a source file (MainWindow.cpp) write coordinates menu button slot, when the coordinates of the button is pressed, coordinates button Groove function , Turned to the coordinates of main interface is displayed. Slot code is "coordinate.showFullScreen ()", that is, full screen coordinate object. Function module interface to jump and other similar.

\section{CNC Interface Design and Development}

Frame Design. The man-machine interface of CNC system frame as shown in Figure 2, displays are mainly divided into four parts: the warning bar and status bar, toolbar, menu bar. Run a program 
status bar includes the trademark or logo, name and location, display, date and time of the current menu; bar is the focus of displays, displays the current File information; alert columns including numerical control system operating status and alarm display; menu bar is mentioned in a class module.

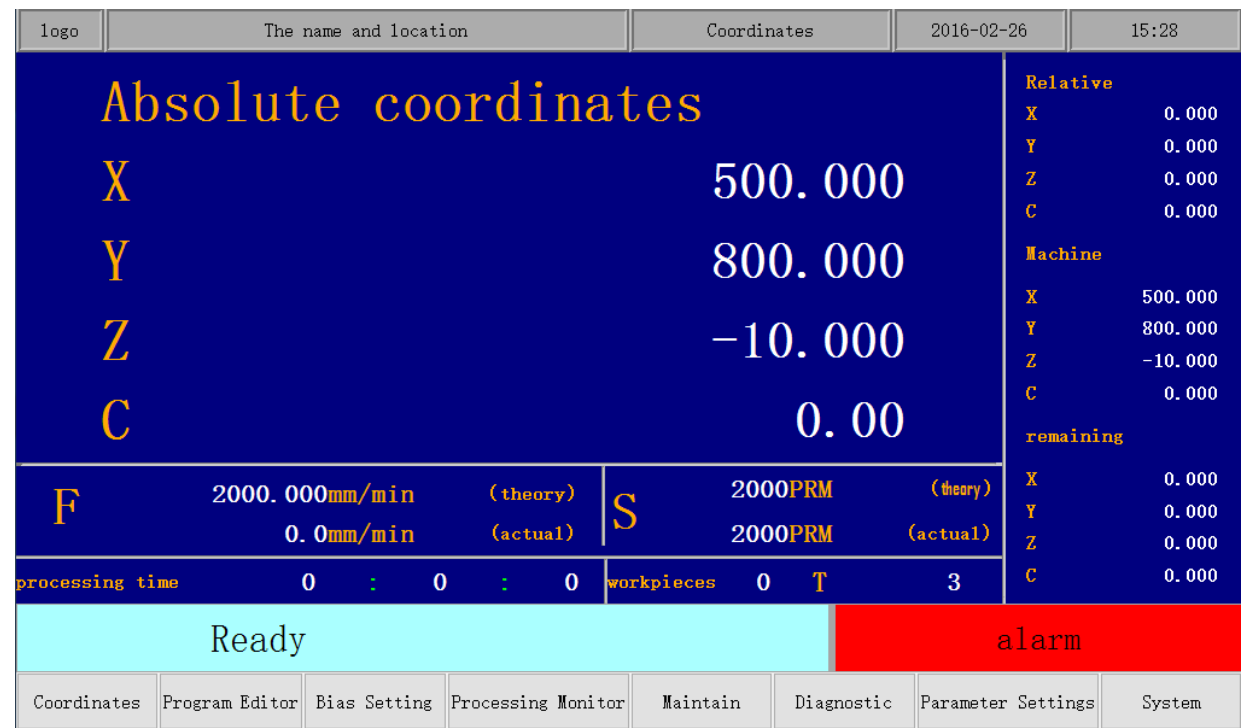

Fig.3. G-code Editor Workflow

G code editor design. Interface design of CNC system is a main feature of the program editor, contains the g-code editor is the core of design. In order to more convenient editing, g-code editor will have the following functions: editing functions, function, features such as auto-completion and syntax detection, workflow, as shown in Figure 3. Editing inherited "QPlainTextEdit" category through the keyboard press the Edit; auto-completion feature inherited "QListWidget" class by querying the library files for code hints and auto-completion feature in the form; grammatical way of detection is the use of regular expressions for line-by-line g-code program checks, use the "QRegExp" class to complete programming. 


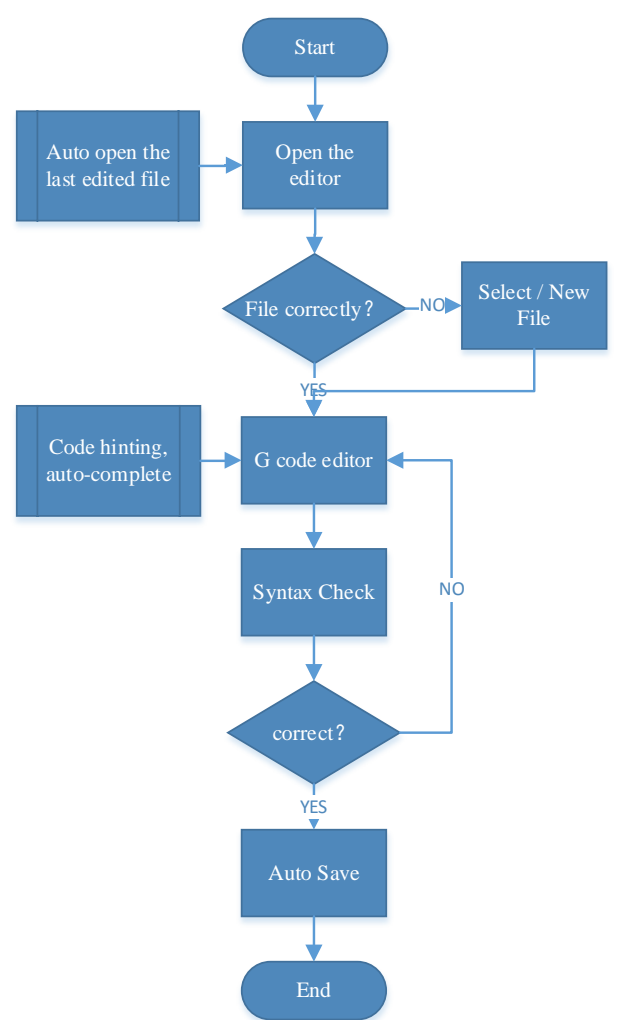

Fig.3. G-code Editor Workflow

File Manager Design. File management capabilities is refers to some basic operations on files, except for some basic new, copy, paste, functions such as open, save, save as, including the choice of location, which involved internal and external storage. Internal storage is a file stored in the local and external storage refers to external storage devices such as an external USB flash drive or portable hard drive. To a USB memory stick, for example, file delivery workflow, as shown in Figure 4. 


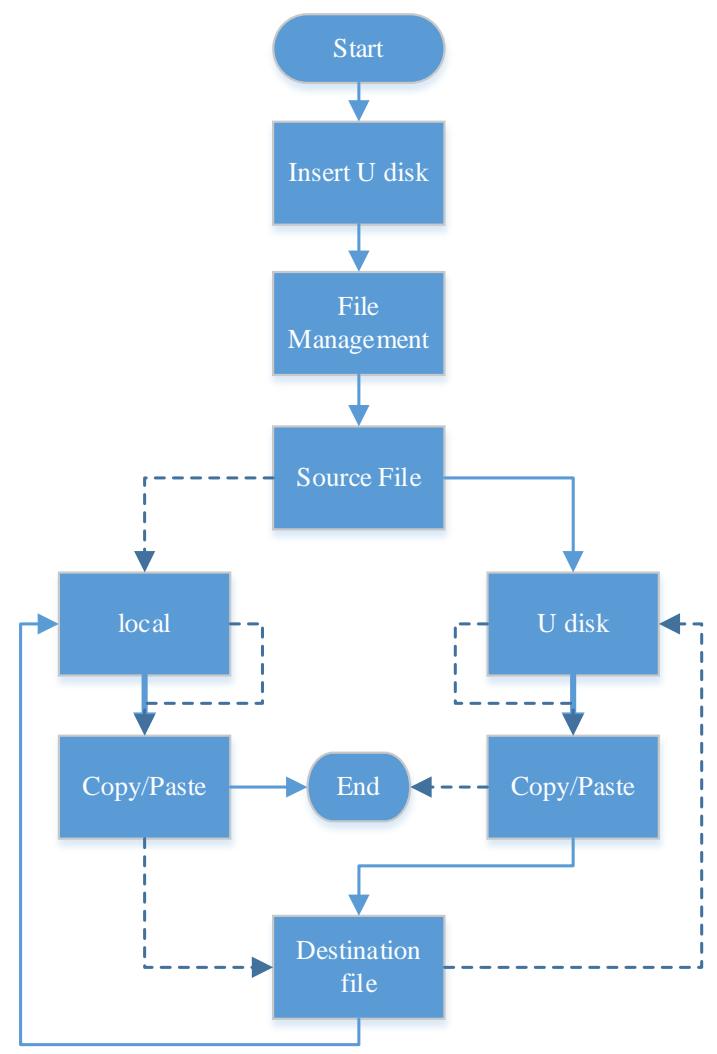

Fig.4. File Delivery Workflow

Graphic Simulation Design. Qt development graphics programs have a big advantage, because "QPainter" class has many drawing functions, including straight lines, arcs, circles, rectangles, and other basic functions. Graphics simulation of numerical control program code are "G00", "G01", "G02" and "G03", "G00" refers to locating straightforward use "moveTo ()" function can be achieved; " G01 "linear interpolation," lineTo () "function can also be done directly; "G02 "and" G03 "means circular interpolation clockwise and counterclockwise circular interpolation, use the draw arc functions" arcTo (a) "implementation, which has three parameters which are bounding rectangle" rectangle ", start angle" startAngle " arc " sweepLength ", but due to the parameters of this function are not so according to the actual situation directly can be discussed later.

Circular interpolation algorithm is shown in Figure 5, dividing the circle into 8, respectively, discussed in the 8-part arc angle and direction, and position of the arc. Programming is based on a known radius of the starting point, end point coordinates and find the " rectangle ", " startAngle " and the " sweepLength " variable values of the three parameters. Algorithm steps are as follows:

(1) Up to the beginning of the coordinates (x1, y1), the ending coordinates (x2, y2) coordinates and RADIUS obtained (x0, y0), calculated according to the following procedure "rectangle".

QRectF rectangle(x0-r, y0-r, 2*r, 2*r);

(2) Calculated according to the formula 1 starting point and the center of the angle between the line and the horizontal line (sharp), " $\alpha$ ", further split into discussions seeking "startAngle" value is "180- $\alpha$ " or " $\alpha$ ".

$$
\alpha=\frac{180}{\pi} \times \operatorname{atan} \frac{y 1-y 0}{x 1-x 0}
$$

(3) According to formula 2 find "sweepLength".

$$
\text { sweepLength }=-2 \times \frac{180}{\pi} \times \operatorname{asin} \frac{\sqrt{(x 2-x 1)^{2}+(y 2-y 1)^{2}}}{2 \times r}
$$


(4) According to "arcTo (rectangle,startAngle,sweepLength)" required to draw graphics.

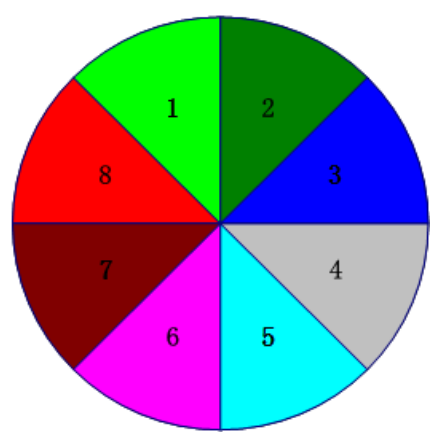

Fig.5. Arc Distribution

\section{Test results}

Interface procedures throughout the system works great, so every little modules to build separate project then consolidated into a major project after the test run. Staging of this paper includes g-code syntax check test section and ARC test, test result is shown in Figure 6. Figure, the "\#300" illegal variable syntax testing question box will pop up showing the first line after the variable is not valid, change and then test the problem goes away, and program syntax and correct; ARC trajectory in the figure is drawn in the g-code order, note arc testing procedures adopted, just sleek largely need to be improved.

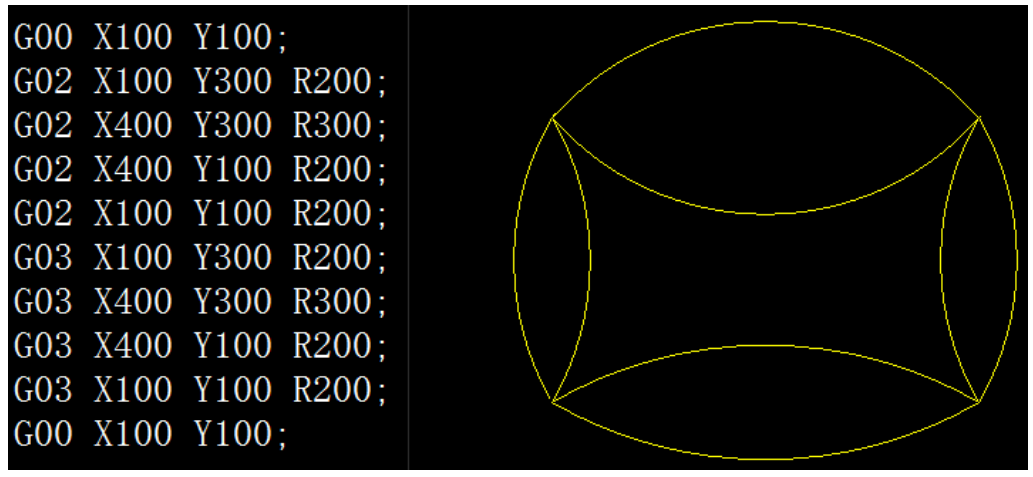

Fig.6. Test Results

\section{Conclusion}

Using the Qt cross-platform programming language design embedded CNC system interface program, through the analysis of system functional requirements and design interface framework and predefined function names, in order to achieve the whole NC interface design. Based on actual experience in the paper gives specific interface development plan, the Qt development workers have 
some significance, but there is still a lot of work for the entire system needs further study, for example, system stability and efficiency of the processes with PLC program running on resource consumption, and so on.

\section{Acknowledgement}

This work was financially supported by the Shandong province's independent innovation and transformation project in 2014, "CNC generation of fieldbus control system application in the field of wood processing machinery industrialization demonstration" (Project No. 2014CGZH0809).

\section{References}

[1] Zheng Hailong. Research of Industrial Control of the Man-machine Interface System Based on Embedded Technology [J]. Computer Measurement \& Control, 2015(3): 959-965.

[2] Zhou Xinlei, Wangwei, et al. Design and Implementation of a Multi-Monitor Display System Based on the Qt for NAOC MUSER Observations [J]. ASTRONOMICAL RESERCH AND TECHNOLOGY, 2015(4): 503-509.

[3] Jiang Bangyou, Yan Xuewen. Based on Embedded Graphical Interface Development and Application [J]. Industrial Control Computer, 2010(6): 29-30.

[4] Su Ming. Research and Implementation on PC-Based Open Control System Application Design Libraries [D]. GuangZhou: South China University of Technology, 2015.

[5]Zhao Yi, You Youpeng. Application of Qt/Embedded in Embedded CNC System [J]. Industrial Control Computer, 2008(4): 68-71. 